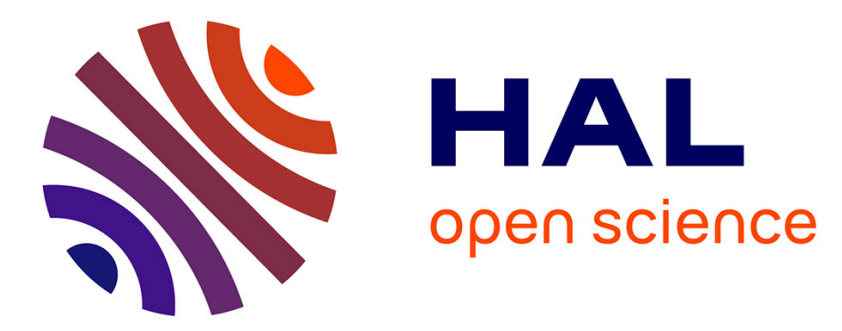

\title{
From Views to Tags Distribution in Youtube
}

Stéphane Delbruel, François Taïani

\section{To cite this version:}

Stéphane Delbruel, François Taïani. From Views to Tags Distribution in Youtube. Middleware'14, Dec 2014, Bordeaux, France. 10.1145/2678508.2678519 . hal-01158149

\section{HAL Id: hal-01158149 \\ https://hal.inria.fr/hal-01158149}

Submitted on 1 Jun 2015

HAL is a multi-disciplinary open access archive for the deposit and dissemination of scientific research documents, whether they are published or not. The documents may come from teaching and research institutions in France or abroad, or from public or private research centers.
L'archive ouverte pluridisciplinaire HAL, est destinée au dépôt et à la diffusion de documents scientifiques de niveau recherche, publiés ou non, émanant des établissements d'enseignement et de recherche français ou étrangers, des laboratoires publics ou privés.

\section{()ㅜ(1)}

Distributed under a Creative Commons Attribution - NonCommercial| 4.0 International 


\section{From Views to Tags Distribution in Youtube}

\author{
Stéphane Delbruel \\ Université de Rennes 1, IRISA \\ stephane.delbruel@irisa.fr
}

\author{
François Taïani \\ Université de Rennes 1, IRISA - ESIR \\ francois.taiani@irisa.fr
}

\begin{abstract}
Better understanding the geographic distribution of user generated content (UGC) can help design better distributed implementations of UGC systems. Although tags play a critical role in many social media, their role in the geographical distribution of UGC content has been little investigated. In this poster, we present some early work analyzing how the tags attached to a video relate to the geographic distribution of a video's views in a Youtube dataset, and hint at how such knowledge might be used to improve current UGC services.
\end{abstract}

\section{General Terms}

Measurement, Algorithm, Design

\section{INTRODUCTION}

Streaming has grown to become one of the largest source of worldwide Internet traffic, with reports of video content accounting for up to $60 \%$ of an ISP's load during peak periods 5]. A large proportion of this traffic is caused by User Generated Content (UGC) services such as Youtube, Dailymotion, or Vimeo: in 2013 for instance, Youtube accounted for $18.69 \%$ of overall network traffic in North America, $28.73 \%$ in Europe, and up to $31.22 \%$ in Asia 11. Storing, processing, and delivering this amount of data poses a constant engineering challenge to both UGC service providers and ISPs. One of the main difficulties lies in the sheer number of submitted videos these systems must process, most of which need to be served to niche audiences, in limited geographic areas 6, 2, 7. Predicting where which video will be viewed is therefore particularly interesting for these systems. Although some earlier works exist on this question, none have - to the best of our knowledge - considered how this problem could be addressed using the tags attached to videos. Tags capture elements of a video's semantic, and therefore provide a particularly promising starting point to analyze how videos with related content may be viewed and distributed geographically.

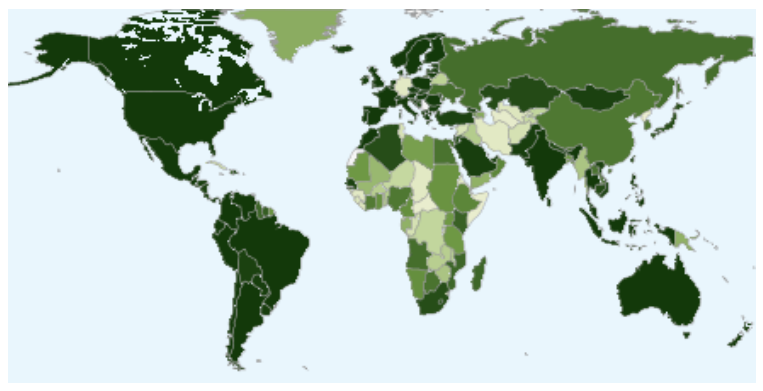

Figure 1: Popularity map of Justin Bieber - Baby ft. Ludacris

In this poster, we present some early results on the relationship between the tags of a video and where this video is viewed, hinting that tags maybe be used as predictive markers of a video's viewing pattern. These results point at interesting research avenues on how UGC systems could be better distributed and improved.

\section{THE DATASET}

We use a YouTube dataset collected in our research group in March 2011 6]. The seed of the dataset are the 10 most popular videos in 25 different countries, obtained through Youtube's public API. The dataset was then completed using a breadth-first snowball sampling of the graph of related videos, as reported by Youtube. For each crawled video, the dataset contains, among others, the video's id, its title, its total number of views, a vector of integers representing the video's popularity by country (the popularity vector for short, more on this below), and a set of descriptive tags provided by the user who uploaded the video [4, 3].

The popularity vector of each video (noted pop $(v)$ ) corresponds to the world map which was provided at the time by Youtube to indicate in which country a video was most popular. Fig. 1 for instance, shows the world map of the video with the most views in our dataset (Justin Bieber Baby ft. Ludacris). Such maps used Google's Map Chart service 1 making it possible to extract for each country an integer - from 0 to 61 -representing the video's popularity in this country.

The original dataset contains 1,063,844 unique videos, but not all videos have a complete set of metadata. For the analysis presented here, we filter out all videos containing

https://developers.google.com/chart/image/docs/ gallery/map_charts 
no tags $(6,736$ videos), or with an incorrect or empty popularity vector. This filtering step results in a dataset with 691,349 videos, associated with 705,415 unique tags, totaling $173,288,616,473$ views.

\section{TOWARDS TAGS' GEODISTRIBUTIONS}

The exact meaning of the popularity vector $\operatorname{pop}(v)$ is unfortunately not documented by Youtube. This vector is however unlikely to capture the proportion of a video's views originating from individual countries. The maximum value by country is caped at 61 , and is more likely to represent a trend in the country. In the video Justin Bieber - Baby ft. Ludacris for instance (Fig. 1), the USA and Singapore have the same value of 61 , although this highly popular video cannot plausibly have been viewed as many times in the USA (pop. $318.5 \mathrm{M}$ ) as in Singapore (pop. 5.4M).

To interpret $\operatorname{pop}(v)$, we take cue from Google Trends ${ }^{2}$, one of the analytics services provided by Youtube's parent company Google, and we consider $\operatorname{pop}(v)[c]$ to represent the intensity of video $v$ in country $c$, i.e. a number proportional to the share of this video's views in this country's Youtube traffic:

$$
\operatorname{pop}(v)[c]=\frac{\operatorname{views}(v)[c]}{\mathbf{y t u b e}[c]} \times K(v)
$$

where views $(v)[c]$ is the number of views of $v$ in country $c$, ytube $[c]$ is the total number of Youtube views in country $c$, and $K(v)$ is a normalization factor, dependent of each video, to scale values in the range $[0-61]$. Neither ytube $[c]$ nor $K(v)$ are available to us. To estimate both, we use the distribution of Youtube traffic provided by Alexa Internet Inc ${ }^{3}$ an authoritative source of Internet traffic, to approximate the distribution of Youtube views per country:

$$
\text { ytube }[c]=\mathbf{p}_{y t}[c] \times T_{y t} \simeq \widehat{\mathbf{p}}_{y t}[c] \times T_{y t}
$$

where $\mathbf{p}_{y t}[c]$ is the proportion of Youtube views in country $c$ at the time our dataset was collected, $T_{y t}$ is the total number of Youtube views at the same time, and $\widehat{\mathbf{p}}_{y t}[c]$ is the Youtube traffic estimated by Alexa for country $c$. Using the total number of views per video (given by our dataset), and the above approximation, we can eliminate $K(v)$ from (1), and reconstruct an approximation of views $(v)$ from $\operatorname{pop}(v)$ and $\widehat{\mathbf{p}}_{y t}$.

To analyze the distribution of tags, we then derive for each unique tag $t$ the number of views associated with $t$ in country $c$ (noted views $(t)[c]$ ), i.e. the aggregated number of views in country $c$ of the videos containing $t$ as tag.

$$
\operatorname{views}(t)[c]=\sum_{v \in \operatorname{videos}(t)} \operatorname{views}(v)[c]
$$

A manual analysis of views $(t)$ reveals that some tags are mainly viewed in particular countries, as the tag favela in Fig. 3. while others are more uniformly distributed, as the tag pop (Fig. 2), the second most viewed tag in our dataset.

This observation leads us to conjecture that the geographic distribution of a video's views might be strongly related to that of its associated tags. In turn, this conjecture suggests that tags might help implement a form of proactive geographic caching, i.e. predicting where a video will be consumed, based on the geographic study of its embodied tags, an avenue we plan to investigate in our future research.

2 http://www.google.com/trends/

${ }^{3}$ http://www .alexa.com/

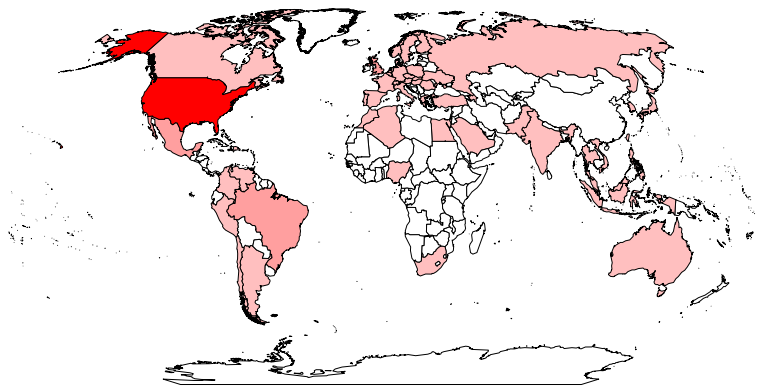

Figure 2: The tag 'pop' tends to follow the world distribution of Youtube users.

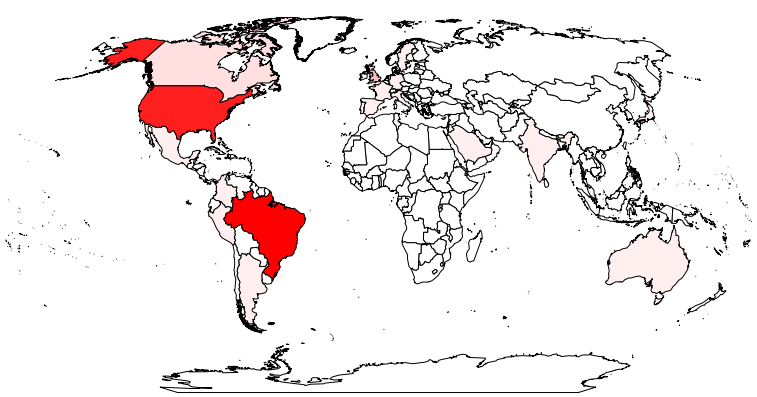

Figure 3: Videos associated with the tag 'favela' are mostly viewed in Brazil

\section{REFERENCES}

[1] Global internet phenomena report: 2h 2013. Technical report, Sandvine Incorporated, 2013.

[2] A. Brodersen, S. Scellato, and M. Wattenhofer. YouTube around the world: Geographic popularity of videos. In $W W W^{\prime} 12$.

[3] G. Geisler and S. Burns. Tagging video: conventions and strategies of the youtube community. In $7 t h$ ACM/IEEE-CS joint Conf. on Digital Libraries, 2007.

[4] S. Greenaway, M. Thelwall, and Y. Ding. Tagging youtube - a classification of tagging practice on youtube. In 12th Int. Conf. on Scientometrics and Informetrics, 2009.

[5] F. Guillemin, B. Kauffmann, S. Moteau, and A. Simonian. Experimental analysis of caching efficiency for youtube traffic in an isp network. In Int Teletraffic Congress, 2013.

[6] K. Huguenin, A.-M. Kermarrec, K. Kloudas, and F. Taïani. Content and geographical locality in user-generated content sharing systems. In NOSSDAV'12. ACM

[7] S. Scellato, C. Mascolo, M. Musolesi, and J. Crowcroft. Track globally, deliver locally: Improving content delivery networks by tracking geographic social cascades. In $W W W^{\prime} 11$. 